\title{
AUTOMATED RECONSTRUCTION OF 3D BUILDINGS IN HISTORIC CITY CENTERS FROM LIDAR DATA AND 2D BUILDING FOOTPRINTS
}

\author{
J. Ingensand ${ }^{1 *}$ M. Nappez ${ }^{1}$, T. Produit ${ }^{1}$, T. Chassin ${ }^{1}$ \\ ${ }^{1}$ GIS-Lab, School of Engineering and Management Vaud, University of Applied Sciences Western Switzerland, Switzerland \\ (jens.ingensand, marion.nappez, timothee.produit, thibaud.chassin)@heig-vd.ch
}

\author{
Commission VI, WG VI/4
}

KEY WORDS: 3D Buildings, Hybrid Reconstruction Methods, Case Study, LIDAR, Cadastral Footprints

\begin{abstract}
:
This paper describes a process for the automated generation of 3D buildings using 2D building footprints derived from cadastral maps and LIDAR point cloud data. In our approach we extract relevant geometric information from 2D building footprints in order to classify point cloud data. One key concept is the fact that roofs in most cases are aligned to the angles of the walls of a building. This concept is utilized to create contiguous surfaces and to extract ridges. In a field study involving two historic city centers in Switzerland we evaluate the results of our approach.
\end{abstract}

\section{INTRODUCTION}

Today the production and utilization of 3D buildings is on the increase. A variety of different usages such as visibility and shadow analyses, energy demand estimation, noise propagation, indoor navigation, infrastructure planning and utility management are possible through the utilization of 3D buildings. (Biljecki et al., 2015). The use of different visualization techniques such as virtual or augmented reality (Goldparvar-Fard et al., 2009) enable the utilization of 3D models in the field by professionals in order to make decisions. Concepts such as BIM (building information modeling) enable architects and engineers to work together on models that include varying information such as the thickness of walls or the position of pipes.

\section{RELATED WORK}

\subsection{Automated reconstruction methods}

Today there are several established approaches to automatically or semi-automatically create 3D models depending on the input data (see figure 1):

- Aerial and terrestrial photographies can be utilized with stereoscopic methods to allow for a creation of 3D building models. In this case different sets of photos from different angles are needed in order to create a 3D model of a building

- LIDAR data: Point clouds usually issued from airborne LIDAR scanning are used for the automatic generation of 3D models

- 2D footprints can be extruded using related information such as the number of floors

Each of the methods has its advantages and disadvantages in terms of precision, complexity or cost. A combination of different methods has been considered by several researchers, e.g.

\footnotetext{
${ }^{*}$ Corresponding author
}

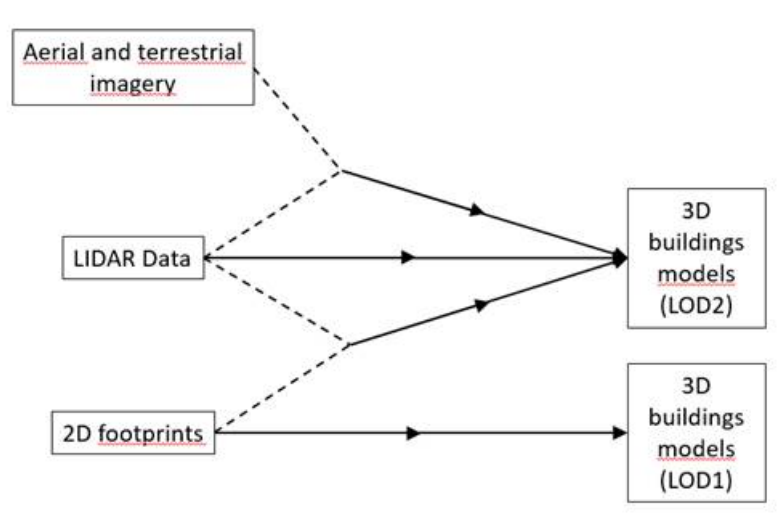

Figure 1. Established approaches for the creation of 3D building models

the utilization of multispectral images (Awrangjeb et al., 2011) (Demir and Baltavias, 2012) together with LIDAR data. Fan et al. (2014) and Teo et al. (2006) have utilized 2D footprints extracted from OpenStreetMap (The OpenStreetmap project, www.openstreetmap.com) for the extraction of points from a LIDAR point cloud to improve the identification of roofs using different algorithms.

Zheng et al. (2017) have utilized geographic directions (e.g. North, North-East, East, etc) for the classification of a TIN (triangulated irregular network) extracted from a LIDAR point cloud in the Indianapolis (USA) urban area. Depending on the geographic direction, the triangles are classified and aggregated in order to form contiguous surfaces. Alexander et al. (2009) have extracted the four main aspects (azimuth angles) from 2D footprints in order to classify the triangles of a TIN in the urban area of Portbury near Bristol (UK).

\subsection{Building morphology}

Buildings are complex and often divided into several subbuildings. Moreover buildings can be attached to other buildings 
and furthermore buildings can be partly or entirely underground. In most scientific papers the detection of the most important part of a building, the roof, is the focus of interest.

According to Zheng et al. (2017) different types of roofs can be distinguished such as:

- Gable roofs

- Hipped roofs

- Half-hipped roofs

- Pyramid roofs

- Cross gable roofs

- Intersecting roofs

Depending on different variables such as culture and history, climate, natural hazards or available space the morphology of buildings can vary considerably. In some places certain types of roofs are more predominant than other types. For instance in Indianapolis where Zheng et al. (2017) carried out their study, streets follow a rectangular pattern and in the central business district modern skyscrapers are the predominant type of buildings. On the other hand the study carried out by Alexander et al. (2009) in Portbury near Bristol shows a pattern of British terraced houses.

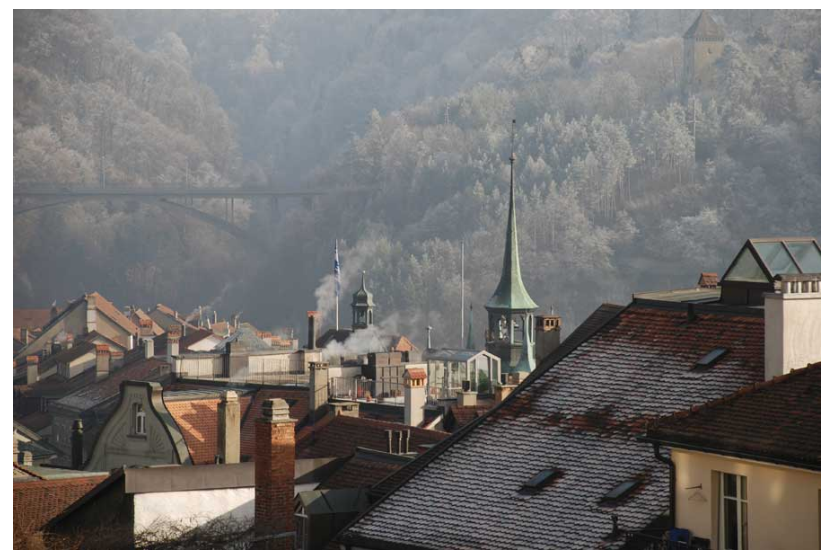

Figure 2. Roofs in the historic city center of Fribourg (C) City of Fribourg Copyright 2017, www.ville-fribourg.ch

The cities of Nyon and Fribourg (see in figure 2), Switzerland, which are the focus of our study, have city centers with buildings dating back to the 12th century. At the time some of these old buildings were built, medieval methods were utilized for the construction of the walls and the roofs. In the course of time, buildings were modified, renovated, etc. Due to these facts the roofs and buildings of a historic city center do not necessarily follow even geometric forms. This is one reason why a historic city center with varying angles and complex structures is more challenging to model using automated methods.

\subsection{Building footprints}

$2 \mathrm{D}$ footprints reflect the complexity of a building to a certain degree as different parts of a building can be distinguished. Moreover 2D footprints indicate the length and the azimuth of each wall segment at the ground level of a building. A roof surface's azimuth is in the vast majority of cases aligned to a corresponding wall. Thereby we consider the azimuth of a wall segment an important element that can be taken into account in order to detect different facets of a roof
In Switzerland the cadastre has a legal value and therefore needs to cover the whole of the country. A numeric cadastre is maintained in most major cities of Switzerland - in some rural regions the cadastre is still based on digitized paper maps. The cadastre reflects the owner of a parcel and the geometric properties of the parcel and the buildings situated on the parcel.

\section{CASE STUDY}

\subsection{Input data}

For the computation of complete 3D buildings we utilized datasets for two different Swiss cities: the city of Fribourg and the city of Nyon. For both cities we utilized two different datasets:

- a LIDAR point-cloud which already included a classification (e.g. vegetation, buildings, etc). For the city of Nyon the resolution was about 30 points per square-meter and for the city of Fribourg the resolution was about 10 points per square-meter

- 2D building footprints extracted from the cadastre

The Swiss cadastre is required to fulfill certain criteria regarding precision. For instance, for the Nyon-dataset the precision is within 30 centimeters. The cadastre is continuously updated by surveying campaigns.

To ensure that the building footprints would match the LIDAR point-cloud from a time-perspective, the cadastral footprints were extracted for the same period of time as the LIDAR-flight.

\subsection{Altitude extraction and segmentation of $2 \mathrm{D}$ footprints}

The creation of 3D buildings makes it necessary to not only detect roofs, but also to generate facades based on 2D footprints and the roofs. For a correct visualization of a building, the facade must start at the lowest point of the building. Therefore, the first step of our automated process is the detection of the minimum altitude based on the lowest point of the LIDAR point cloud intersecting with the $2 \mathrm{D}$ footprint. The $2 \mathrm{D}$ polygons were thereafter converted to $3 \mathrm{D}$ polygons using the minimum altitude.

In order to utilize 2D footprints for the detection of roof surfaces it is important that the footprints already reflect the division of a building into sub-buildings. A sub-building is for instance a part of a building with a different type of roof (e.g. main building: gable roof, appendix: flat roof) or a part that shows a significant difference in height. To accomplish this segmentation, several methods can be utilized such as image processing techniques; e.g. the Canny edge detection described by Novacheva (2008). One problem with these image processing techniques is that the detected breaklines might not match the exact position of the underlying walls. Another potential problem is the fact that two adjacent flat surfaces with different altitudes might not (depending on the position of the sun at the time the image was taken) show any significant difference in color and thus the breakline between the two surfaces would not be detected.

In our approach we have chosen to utilize LIDAR data together with $2 \mathrm{D}$ footprints to identify the breaklines that will be used for the segmentation of the footprints. An inspiration for this process was Vosselman and Dijkman's article on the detection of height-jumps (Vosselman and Dijkman, 2001). A first step in this process is to identify zones in the LIDAR data which show considerable differences in height using the following steps: 
1. One point of the point-cloud is selected

2. All neighboring points within a certain distance (threshold depends on the density of the point cloud) are selected and heights are compared

3. If the difference in height is greater than a certain threshold (e.g. 1 meter) the point can be considered as being part of a breakline

4. If several points within a certain distance to other points of the point cloud show this difference in height, these points can be considered as being part of a larger zone which itself can be considered as a breakline-zone

5. A convex hull including the identified points forms a breakline-zone

In order to cut the 2D footprints using these breakline-zones two different rules are applied:

1. in the $2 \mathrm{D}$ footprint concave angles are identified. The angle-composing lines are simply drawn further until they intersect with a wall on the opposite side of the 2D footprint. If the created lines coincide with the breakline-zone to a certain percentage, (e.g. 90\%) the line can be utilized to cut the $2 \mathrm{D}$ footprint into smaller pieces. This allows for a precise identification of the line where the footprint needs to be cut (see figure 3 )

2. if a breakline-zone does not coincide with a line, it is possible to identify its medial axis and to utilize this axis in order to cut the $2 \mathrm{D}$ footprint. In this case the calculated axis must either form a polygon within the $2 \mathrm{D}$ footprint or cut the 2D footprint into two parts

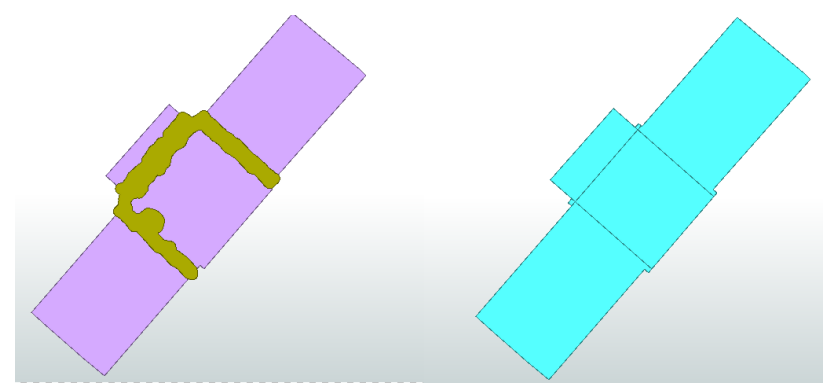

Figure 3. Segmentation of the 2D footprint using concave angles. On the left: breakline-zones identified using LIDAR data. On the right: lines created as a continuation of the lines creating concave angles

\subsection{Roof detection}

3.3.1 Flat roofs For the determination of flat roofs a TIN is generated using the LIDAR point cloud intersected with each part of a segmented 2D footprint. For each triangle of the TIN the slope is calculated. If a certain percentage of the triangles show very weak slopes (e.g. 15 degrees) then the roof of the segmented footprint is considered as flat.

3.3.2 Complex roofs The generation of complex (i.e. not flat) roofs is based on the idea that each surface of the roof has a corresponding wall. Therefore, the $2 \mathrm{D}$ footprint is first decomposed into separate lines. For each of the lines, the azimuth is calculated. Thereafter, all available azimuths are automatically assembled in a table. Alexander et al. (2009) use a similar method, however only the four main angles are conserved. In our case, the disassembled walls of a cadastral footprint can have up to 3040 azimuth angles. We therefore reduce the number of available azimuths by matching similar azimuths. (e.g. 87 degrees and 89 degrees can be matched to 88 degrees). This allows us to identify the main azimuths of a building.

The TIN's of the roofs which have not been identified as being flat roofs are disassembled into triangles. For each triangle, both the azimuth and the slope are calculated. The slope helps us to eliminate flat and very steep triangles which for instance may be the result of a breakline. The resting triangles are classified using the main azimuth values identified from the cadastral footprints. With the classified triangles it becomes possible to dissolve the triangles to contiguous surfaces (see figure 4). With a threshold value which depends on the density of the point cloud, surfaces with a small calculated area can be eliminated.

The assembled surfaces are then utilized to identify points where two surfaces touch each other. Through these touching points ridge lines can be drawn. The cadastral footprint is thereafter draped on top of these ridge lines.

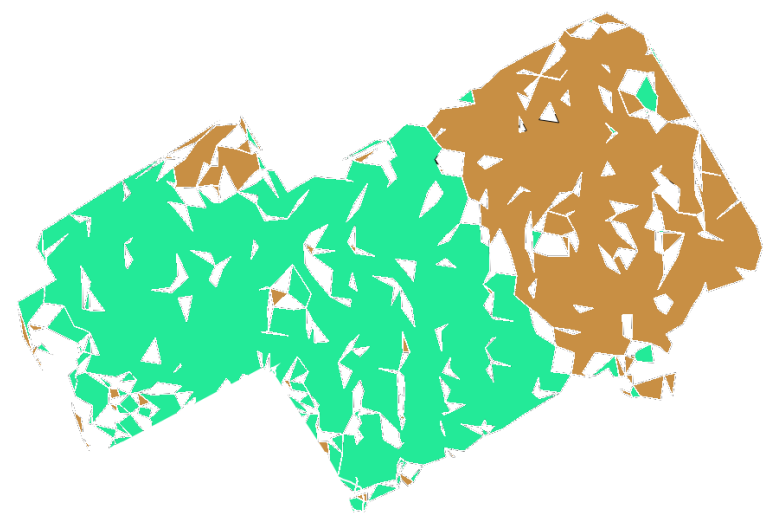

Figure 4. Classification of triangles according to the azimuth angles extracted from a 2D footprint and aggregation of the triangles to contiguous surfaces based on the azimuth classification

3.3.3 Facades The facades are created using the identified roofs and the matching cadastral footprints which were previously put in 3D using the minimum altitude identified from the point cloud. Thereafter, the roofs are extruded downwards and the cadastral footprints are extruded upwards. The two resulting solid shapes are clipped and the shapes that are inclined 90 degrees with respect to the soil are extracted as facades.

\section{RESULTS}

Our process (see figure 5) enabled us to create 3D city models of both cities: Nyon and Fribourg. Several difficulties were encountered:

- Some 2D footprints from the cadastre were either partially or entirely subterranean buildings (e.g. car parks). These buildings were either modeled as flat roofs or, if there were no classified points, not created at all

- Sometimes two or several physical buildings were attached to each other and represented by a single polygon. 
This occurred if the owner of both buildings was the same. In this case the segmentation of the cadastre enabled us to separate the buildings

- In many cases a roof of one building protruded an adjacent building. In order to eliminate these surfaces two measures were taken: 1 . once a surface had been assembled a ratio (surface / circumference) was calculated to identify and eliminate very narrow surfaces. 2. a fixed buffer eliminated ridge lines that were too close

- It occurred that other objects such as for instance bridges that were constructed over a building covered the buildings underneath. In this case the generation of the roof either failed or resulted in an erroneous surface

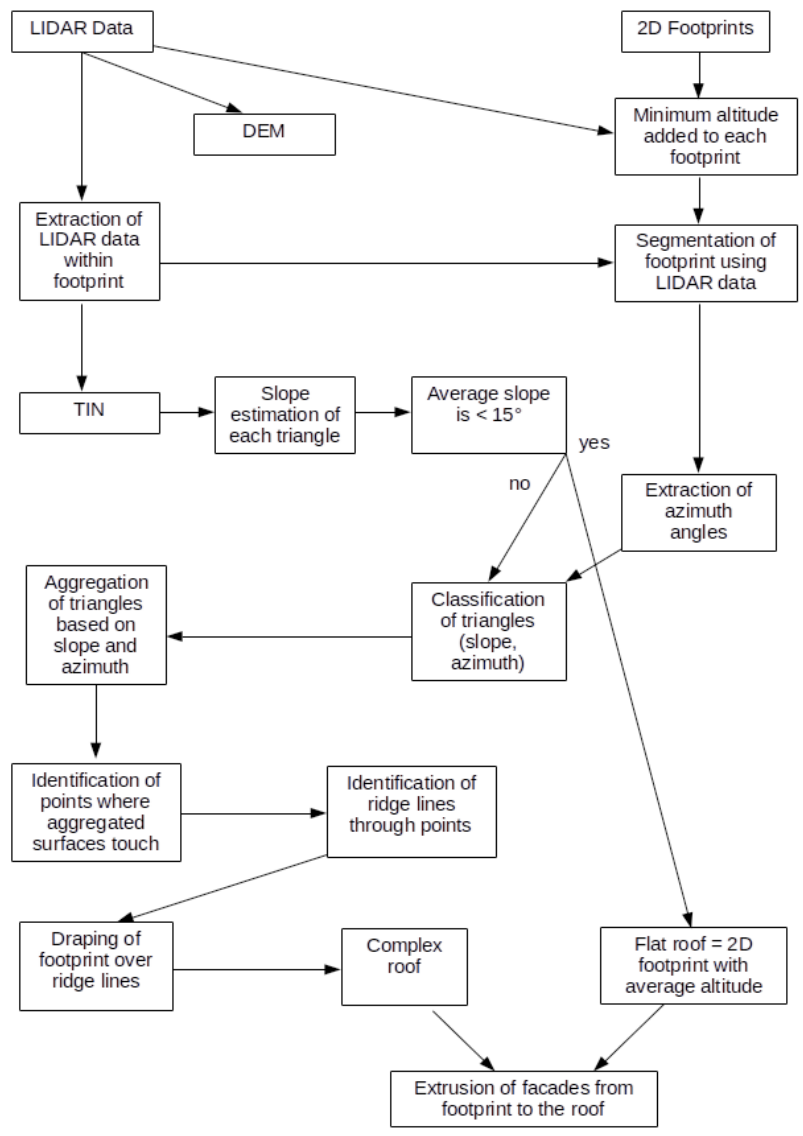

Figure 5. Processing schema. The input data is a LIDAR point cloud and 2D footprints derived from the cadastre. The output is a DEM (raster) as well as building roofs and facades

The process always required some calibration regarding the input data. However once it was calibrated the process was able to automatically generate 5'000 buildings in the city of Fribourg and about 10'000 - 10'500 buildings in the city of Nyon (see figure 6) and surrounding villages (counted using the number of cadastral footprints).

Awnings (i.e. roofs that span farther than the 2D footprint) were not considered at all in our process due to the fact that the point cloud data is automatically cut with the $2 \mathrm{D}$ footprint.

\section{CONCLUSIONS AND PERSPECTIVES}

Today the utilization of virtual 3D city models within different contexts and for varying purposes is on the increase. Therefore

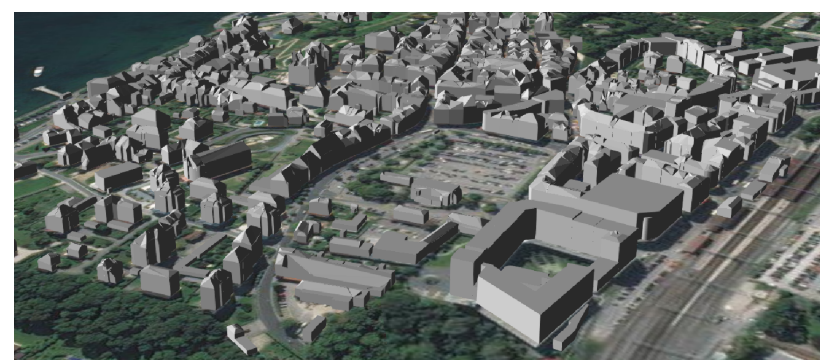

Figure 6. Automatically generated buildings for the city of Nyon

automated methods are an important tool for the generation of this data.

Historic city centers are a difficult terrain for automated methods due to the fact that buildings in many cases have uneven roof surfaces and are attached to each other.

Our case study showed that $2 \mathrm{D}$ footprints are a valuable support for the automated generation of 3D buildings from LIDAR data. However the definition of what can be considered as a building can differ between footprints and a classified point cloud. 2D footprints issued from the cadastre can for instance describe buildings that are partially or entirely underground. Another point is that a cadastral footprint can correspond to two or more physical buildings if buildings are attached to each other and if the buildings are owned by the same person. This problem frequently occurs in historic city centers.

Several researchers have had the idea to match the azimuths of the triangles of a TIN in order to assemble surfaces. These casestudies, however, were conducted in environments that were very different to a historic central European city center. Therefore the segmentation of the cadastre using LIDAR data is an important step in order to distinguish buildings and roof surfaces.

Our method has the advantage that the size of the surfaces to be detected can be adapted depending on the density and quality of the point cloud - on the other hand the generated buildings are not perfect. In some places the density of the point cloud was not satisfying since other objects such as bridges covered the buildings

Moreover it can be regarded as a disadvantage that awnings are not considered if the goal is to display the generated 3D buildings in a virtual environment. On the other hand, the lack of awnings makes it easier to utilize the 3D buildings for $3 \mathrm{D}$ printing.

Although the process required an initial calibration phase, we were able to automatically generate large quantities of data. An idea for future work is therefore the automation of this calibration phase depending on the density and quality of the point cloud and the precision of the cadastral footprints. Especially for the identification of some parameters (e.g. fixed thresholds), statistical methods such as k-means can be utilized.

The fact that two different datasets (footprints and LIDAR data) were utilized made it possible to mutually improve the quality of both data-sets. On one hand, 2D footprints can be improved using LIDAR data (segmentation, analysis whether classified points from the point cloud are within a 2D footprint), on the other hand the utilization of LIDAR data can be improved using 2D footprints (splitting of LIDAR data with 2D footprints and identification of azimuth angles). Moreover, the splitting of the LIDAR 
data using 2D footprints also had the advantage of decreasing processing time due to the fact that only buildings were analyzed and not the point cloud as a whole. Another advantage of our process is that an unclassified point cloud theoretically could be utilized since the process basically cuts all points from the point cloud that are within the cadastral footprint.

Today many different methods exist for the automatic or semiautomatic creation of buildings. These methods were tested in very different environments with quite various data. We suggest that an idea for further research is to identify which method fits best with which type of roof morphology, with respect to the datasets that are available. A first step in this direction would be the establishment of ground truth 3D buildings with corresponding datasets and the elaboration of indicators to quantitatively compare the performance of various methods.

Our method provides a snapshot of a city at the time of the LIDAR acquisition flight. However, cities are continuously evolving and the usage of $3 \mathrm{D}$ city models for different goals is on the increase. A main challenge is therefore to identify ways to continuously correct and update a $3 \mathrm{D}$ city model. The classic approach would be to simply conduct a new LIDAR flight, but this is very costly. Several other approaches could be utilized:

- Crowdsourcing proves to be a working concept for 2D data in for instance the OpenStreetMap project. The utilization of volunteers to improve or generate $3 \mathrm{D}$ data is a more recent method. Zipf and Uden (2012) for instance have developped a concept that enables citizen to share 3D data using a crowdsourcing platform

- With the democratization of drones which are yet able to embark both a camera or a LIDAR scanner, a massive quantity of 3D data and textures at local scale can be generated

- The increasing utilization of BIM also raises the production of precise $3 \mathrm{D}$ building models. However a common problem is to integrate new buildings in existing $3 \mathrm{D}$ city models due to georeferencing issues

The resulting challenge is to gather all these efforts and models of various quality, generated by private and public entities and stored in various places, in a single platform.

\section{ACKNOWLEDGEMENTS}

The authors would like to thank the cities of Fribourg and Nyon for their support regarding this project.

\section{REFERENCES}

Alexander, C., Smith-Voysey, S., Jarvis, C. and Tansey, K., 2009. Integrating building footprints and LiDAR elevation data to classify roof structures and visualise buildings. Computers, Environment and Urban Systems 33(4), pp. 285 - 292.

Awrangjeb, M., Zhang, C. and Fraser, C. S., 2011. Automatic Reconstruction of Building Roofs Using LIDAR and Multispectral Imagery. In: International Conference on Digital Image Computing: Techniques and Applications.

Biljecki, F., Stoter, J., Ledoux, H., Zlatanova, S. and Coltekin, A., 2015. Applications for 3D City Models: State of the Art Review. ISPRS International Journal of Geo-Information 2015(4), pp. 2842-2889.
Demir, N. and Baltavias, E., 2012. Automated Modeling of 3D Building Roofs Using Image and LIDAR Data. In: 22nd ISPRS Congress (ed.), 22nd ISPRS Congress, Melbourne, Australia.

Fan, H., Yao, W. and Fu, Q., 2014. Segmentation of Sloped Roofs from Airborne LiDAR Point Clouds Using Ridge-Based Hierarchical Decomposition. Remote Sensing 6, pp. 3283-3301.

Goldparvar-Fard, M., Feniosky, P. and Savares, S., 2009. D4AR A 4-Dimensional augmented reality model for automating construction progress monitoring data collection, processing and communication. Journal of Information Technology in Construction 14, pp. 129-153.

Novacheva, A., 2008. Building Roof Reconstruction from LIDAR Data and Aerial Images through Plane Extraction and Colour Edge Detection. The International Archives of the Photogrammetry, Remote Sensing and Spatial Information Sciences XXXVII(Part B6b), pp. 53-57.

Teo, T.-A., Rau, J.-Y., Chen, L.-C., Liu, J.-K. and Hsu, W.-C., 2006. Reconstruction of Complex Buildings using LIDAR and 2D Maps. Springer, Berlin, Heidelberg, pp. 345-354.

Vosselman, G. and Dijkman, S., 2001. 3D Building Model Reconstruction from Point Clouds and Ground Plans. International Archives of Photogrammetry and Remote Sensing.

Zheng, Y., Weng, Q. and Zheng, Y., 2017. A Hybrid Approach for Three-Dimensional Building Reconstruction in Indianapolis from LiDAR Data. Remote Sensing.

Zipf, A. and Uden, M., 2012. OpenBuildingModels - Towards a platform for crowdsourcing virtual 3D cities. In: 7th 3D GeoInfo Conference, Quebec City, QC, Canada (2012). 